Homology, Homotopy and Applications, vol.11(2), 2009, pp.229-250

\title{
REMARKS ON FINITE SUBSET SPACES
}

\author{
SADOK KALLEL AND DENIS SJERVE
}

(communicated by J. Daniel Christensen)

\begin{abstract}
This paper expands on and refines some known and less well-known results about the finite subset spaces of a simplicial complex $X$ including their connectivity and manifold structure. It also discusses the inclusion of the singletons into the threefold subset space and shows that this subspace is weakly contractible but generally non-contractible unless $X$ is a cogroup. Some homological calculations are provided.
\end{abstract}

\section{Statement of results}

Let $X$ be a topological space (always assumed to be path-connected), and $k$ a positive integer. It has become increasingly useful in recent years to study the space

$$
\operatorname{Sub}_{n} X:=\left\{\left\{x_{1}, \ldots, x_{\ell}\right\} \subset X \mid \ell \leqslant n\right\}
$$

of all finite subsets of $X$ of cardinality at most $n[\mathbf{1}, \mathbf{3}, \mathbf{9}, \mathbf{1 5}, \mathbf{1 9}, \mathbf{2 3}]$. This space is topologized as the identification space obtained from $X^{n}$ by identifying two $n$-tuples if and only if the sets of their coordinates coincide [4]. The functors $\operatorname{Sub}_{n}(-)$ are homotopy functors in the sense that if $X \simeq Y$, then $\operatorname{Sub}_{n}(X) \simeq \operatorname{Sub}_{n}(Y)$. If $k \leqslant n$, then $\operatorname{Sub}_{k} X$ naturally embeds in $\operatorname{Sub}_{n} X$. We write $j_{n}: X \hookrightarrow \operatorname{Sub}_{n} X$ for the inclusion given by $j_{n}(x)=\{x\}$.

This paper takes advantage of the close relationship between finite subset spaces and symmetric products to deduce a number of useful results about them.

As a starting point, we discuss cell structures on finite subset spaces. We observe in Section 3 that if $X$ is a finite $d$-dimensional simplicial complex, then $\operatorname{Sub}_{n} X$ is an $n d$-dimensional CW-complex and of which $\mathrm{Sub}_{k} X$ for $k \leqslant n$ is a subcomplex (Proposition 3.1). Furthermore, Sub $X:=\coprod_{n \geqslant 1} \operatorname{Sub}_{n} X$ has the structure of an abelian CWmonoid (without unit) whenever $X$ is a simplicial complex.

In Section 4 we address a connectivity conjecture stated in [25]. We recall that a space $X$ is $r$-connected if $\pi_{i}(X)=0$ for $i \leqslant r$. A contractible space is $r$-connected for all positive $r$. In [25] Tuffley proves that $\operatorname{Sub}_{n} X$ is $n-2$-connected and conjectures that it is $n+r-2$-connected if $X$ is $r$-connected. We are able to confirm his conjecture for the three-fold subset spaces. In fact we show

Research supported by NSERC grant A7218.

Received March 2, 2009, revised July 26, 2009; published on November 29, 2009.

2000 Mathematics Subject Classification: 55U10, 55S15.

Key words and phrases: finite subsets, connectivity, homology, manifold structure.

This article is available at http://intlpress.com/HHA/v11/n2/a12

Copyright (c) 2009, International Press. Permission to copy for private use granted. 
Theorem 1.1. If $X$ is $r$-connected, $r \geqslant 1$ and $n \geqslant 3$, then $\operatorname{Sub}_{n} X$ is $r+1$-connected.

In Section 5 we address a somewhat surprising fact about the embeddings

$$
\operatorname{Sub}_{k} X \hookrightarrow \operatorname{Sub}_{n} X, \quad k \leqslant n .
$$

A theorem of Handel [9] asserts that the inclusion $j: \operatorname{Sub}_{k}(X) \hookrightarrow \operatorname{Sub}_{2 k+1}(X)$ for any $k \geqslant 1$ is trivial on homotopy groups (i.e. "weakly trivial"). This is, of course, not enough to conclude that $j$ is the trivial map, and in fact it need not be. Let $\operatorname{Sub}_{k}\left(X, x_{0}\right)$ be the subspace of $\operatorname{Sub}_{k} X$ of all finite subsets containing the basepoint $x_{0} \in X$. Handel's result is deduced from the more basic fact that the inclusion $j_{x_{0}}: \operatorname{Sub}_{k}\left(X, x_{0}\right) \hookrightarrow \operatorname{Sub}_{2 k-1}\left(X, x_{0}\right)$ is weakly trivial. The following theorem implies that these maps are often not null-homotopic.

Theorem 1.2. The embeddings

$$
j_{x_{0}}: X \hookrightarrow \operatorname{Sub}_{3}\left(X, x_{0}\right), \quad x \mapsto\left\{x, x_{0}\right\}
$$

and

$$
j: X \hookrightarrow \operatorname{Sub}_{3}(X), \quad x \mapsto\{x\},
$$

are both null-homotopic if $X$ is a cogroup. If $X=S^{1} \times S^{1}$ is the torus, then both $j$ and $j_{x_{0}}$ are non-trivial in homology and are hence essential.

For a definition of a cogroup, see Section 5. In particular, suspensions are cogroups. The second half of Theorem 1.2 follows from a general calculation given in Section 5 which exhibits a model for $\operatorname{Sub}_{3}\left(X, x_{0}\right)$ and uses it to show that its homology is an explicit quotient of the homology of the symmetric square $\mathrm{SP}^{2} X$ by a submodule determined by the coproduct on $H_{*}(X)$. One deduces, in particular, a homotopy equivalence between $\operatorname{Sub}_{3}\left(\Sigma X, x_{0}\right)$ and the reduced symmetric square $\overline{\mathrm{SP}}^{2}(\Sigma X)$ (cf. Section 2.1 and Proposition 5.6). The methods in Section 5 are taken up again in [12] where an explicit spectral sequence is devised to compute $H_{*}\left(\operatorname{Sub}_{n} X\right)$ for any finite simplicial complex $X$ and any $n \geqslant 1$.

The final two sections of this paper deal with manifold structures on $\operatorname{Sub}_{n} X$ and top homology groups. It is known that $\mathrm{Sub}_{2} X=\mathrm{SP}^{2} X$ is a closed manifold if and only if $X$ is closed of dimension 2 . This is a consequence of the fact that $\mathrm{SP}^{2}\left(\mathbb{R}^{d}\right)$ is not a manifold if $d>2$, while $\mathrm{SP}^{2}\left(\mathbb{R}^{2}\right) \cong \mathbb{R}^{4}[\mathbf{2 0}]$. The following complete description is due to Wagner [26]:

Theorem 1.3. Let $X$ be a closed manifold of dimension $d \geqslant 1$. Then $\operatorname{Sub}_{n} X$ is a closed manifold if and only if either

(i) $d=1$ and $n=3$, or

(ii) $d=2$ and $n=2$.

This result is established in Section 7 where we use, in the case $d \geqslant 2$, the connectivity result of Theorem 1.1, one observation from [17] and some homological calculations from [13]. In the case $d=1$, we reproduce Wagner's cute argument. Furthermore in that section, we refine a result of Handel's [9] on the top homology groups of $\operatorname{Sub}_{n} X$ when $X$ is a manifold. We point out that if $X$ is a closed orientable 
manifold of dimension $d \geqslant 2$, then the top homology group $H_{n d}\left(\operatorname{Sub}_{n} X\right)$ is trivial if $d$ is odd and is $\mathbb{Z}$ if $d$ is even. This group is always trivial if $X$ is not orientable (see Section 6).

\section{Acknowledgements}

This work was initiated at PIMS in Vancouver and the first author would like to thank the institute for its hospitality.

\section{Basic constructions}

All spaces $X$ in this paper are path-connected, paracompact, and have a chosen basepoint $x_{0}$.

The way we will think of $\operatorname{Sub}_{n} X$ is as a quotient of the $n$-th symmetric product $\mathrm{SP}^{n} X$. This symmetric product is the quotient of $X^{n}$ by the permutation action of the symmetric group $\mathfrak{S}_{n}$. The quotient map $\pi: X^{n} \longrightarrow \operatorname{SP}^{n} X$ sends $\left(x_{1}, \ldots, x_{n}\right)$ to the equivalence class $\left[x_{1}, \ldots, x_{n}\right]$. It will be useful sometimes to write such an equivalence class as an an abelian product $x_{1} \cdots x_{n}, x_{i} \in X$. There are topological embeddings

$$
j_{n}: X \hookrightarrow \mathrm{SP}^{n} X, \quad x \mapsto x x_{0}^{n-1} .
$$

The finite subset space $\operatorname{Sub}_{n} X$ is obtained from $\mathrm{SP}^{n} X$ through the identifications

$$
\left[x_{1}, \ldots, x_{n}\right] \sim\left[y_{1}, \ldots, y_{n}\right] \Longleftrightarrow\left\{x_{1}, \ldots, x_{n}\right\}=\left\{y_{1}, \ldots, y_{n}\right\} .
$$

In multiplicative notation, elements of $\operatorname{Sub}_{n} X$ are products $x_{1} x_{2} \cdots x_{k}$ with $k \leqslant n$, and subject to the identifications $x_{1}^{2} x_{2} \cdots x_{k} \sim x_{1} x_{2} \cdots x_{k}$.

The topology of $\mathrm{Sub}_{n} X$ is the quotient topology inherited from $\mathrm{SP}^{n} X$ or $X^{n}[\mathbf{9}]$. When $X$ is Hausdorff, this topology is equivalent to the so-called Vietoris finite topology whose basis of open sets are sets of the form

$$
\left[U_{1}, \ldots, U_{k}\right]:=\left\{S \in \operatorname{Sub}_{n} X \mid S \subset \bigcup_{i=1}^{k} U_{i} \text { and } S \cap U_{i} \neq \emptyset \text { for each } i\right\},
$$

where $U_{i}$ is open in $X$ [26]. When $X$ is a metric space, $\operatorname{Sub}_{k} X$ is again a metric space under the Hausdorff metric, and hence it inherits a third and equivalent topology [26]. In all cases, for any topology we use, continuous maps between spaces induce continuous maps between their finite subset spaces.

Example 2.1. Of course $\mathrm{Sub}_{1} X=X$ and $\mathrm{Sub}_{2} X=\mathrm{SP}^{2} X$. Generally, if $\boldsymbol{\Delta}^{n+1} X \subset$ $\mathrm{SP}^{n+1} X$ denotes the image of the fat diagonal in $X^{n+1}$, that is

$$
\boldsymbol{\Delta}^{n+1} X:=\left\{x_{1}^{i_{1}} \ldots x_{r}^{i_{r}} \in \mathrm{SP}^{n+1} X \mid r \leqslant n, \sum i_{j}=n+1 \text { and } i_{j}>0\right\},
$$

then there is a map

$$
q: \boldsymbol{\Delta}^{n+1} X \longrightarrow \operatorname{Sub}_{n} X, \quad x_{1}^{i_{1}} \ldots x_{r}^{i_{r}} \longrightarrow\left\{x_{1}, \ldots, x_{r}\right\}
$$


and a pushout diagram

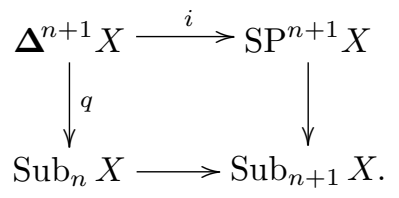

This is quite clear since we obtain $\operatorname{Sub}_{n+1} X$ by identifying points in the fat diagonal to points in $\operatorname{Sub}_{n} X$. In particular, when $n=2$, we have the pushout

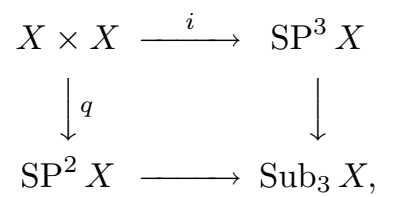

where $q(x, y)=x y$ and $i(x, y)=x^{2} y$. The homology of $\operatorname{Sub}_{3}(X)$ can then be obtained from a Mayer-Vietoris sequence. Some calculations for the three-fold subset spaces are in Section 5.

There are two immediate and non-trivial consequences of the above pushouts. Albrecht Dold shows in [7] that the homology of the symmetric products of a CWcomplex $X$ only depends on the homology of $X$. The pushout diagram in (2) shows that, in the case of the finite subset spaces, this homology also depends on the cohomology structure of $X$. This general fact for the three- and four-fold subset spaces is further discussed in $[\mathbf{2 2}]$.

The second consequence of (2) is that it yields an important corollary.

Corollary 2.2. $\operatorname{Sub}_{n} X$ is simply connected for $n \geqslant 3$.

Proof. We use the following known facts about symmetric products: $\pi_{1}\left(\mathrm{SP}^{n} X\right) \cong$ $H_{1}(X ; \mathbb{Z})$ whenever $n \geqslant 2$, and the inclusion $j_{n}: X \hookrightarrow \mathrm{SP}^{n} X$ induces the abelianization map at the level of fundamental groups. (P.A. Smith $[\mathbf{2 1}]$ proves this for $n=2$, but his argument applies for $n>2[\mathbf{2 2}]$.) For $n \geqslant 3$, consider the composite

$$
X \stackrel{\alpha}{\rightarrow} \Delta^{n} X \stackrel{i}{\rightarrow} \mathrm{SP}^{n} X
$$

with $\alpha(x)=\left[x, x_{0}, \ldots, x_{0}\right]$. The induced map $j_{n *}=i_{*} \circ \alpha_{*}$ on $\pi_{1}$ is surjective, as we pointed out, and hence so is $i_{*}$. Assume we know that $\pi_{1}\left(\operatorname{Sub}_{3}(X)\right)=0$. Then the fact that $i_{*}$ is surjective implies immediately, by the Van-Kampen theorem and the pushout diagram in (2), that $\pi_{1}\left(\operatorname{Sub}_{4} X\right)=0$. By induction, we see that $\pi_{1}\left(\operatorname{Sub}_{n} X\right)$ $=0$ for larger $n$. Therefore, we need only establish the claim for $n=3$. For that we apply Van Kampen to diagram (3). Consider the maps

$$
\tau: x_{0} \times X \hookrightarrow X \times X \stackrel{i}{\rightarrow} \mathrm{SP}^{3} X
$$

and

$$
\beta: X \times x_{0} \rightarrow X \times X \stackrel{q}{\rightarrow} \mathrm{SP}^{2} X .
$$

Now $i(x, y)=x^{2} y$ so that $\tau\left(x_{0}, x\right)=x_{0}^{2} x=j_{3}(x)$ and $\beta\left(x, x_{0}\right)=x x_{0}=j_{2}(x)$. Since the $j_{k}$ 's are surjective on $\pi_{1}$ it follows that $\tau$ and $\beta$ are surjective on $\pi_{1}$. Therefore, 
for any classes $u \in \pi_{1}\left(\mathrm{SP}^{3} X\right)$ and $v \in \pi_{1}\left(\mathrm{SP}^{2} X\right), \exists$ a class $w \in \pi_{1}(X \times X)$ such that $i_{*}(w)=u$ and $q_{*}(w)=v$. This shows that $\pi_{1}\left(\operatorname{Sub}_{3} X\right)=0$.

This corollary also follows from $\left[\mathbf{5 , 2 5}\right.$, where it is shown that $\operatorname{Sub}_{n} X$ is $(n-2)$ connected for $n \geqslant 3$. However, the proof above is completely elementary.

\subsection{Reduced constructions}

For the spaces under consideration, the natural inclusion $\mathrm{Sub}_{n-1} X \subset \mathrm{Sub}_{n} X$ is a cofibration [9]. We write $\overline{\operatorname{Sub}}_{n} X:=\operatorname{Sub}_{n} X / \operatorname{Sub}_{n-1} X$ for the cofiber. Similarly, $\mathrm{SP}^{n-1} X$ embeds in $\mathrm{SP}^{n} X$ as the closed subset of all configurations $\left[x_{1}, \ldots, x_{n}\right]$ with $x_{i}$ at the basepoint for some $i$. We set $\overline{\mathrm{SP}}^{n} X:=\mathrm{SP}^{n} X / \mathrm{SP}^{n-1} X$, the symmetric smash product.

Note that even though $\mathrm{SP}^{2} X$ and $\mathrm{Sub}_{2} X$ are the same, there is an essential difference between their reduced analogs. The difference here comes from the fact that the inclusion $X \hookrightarrow \operatorname{Sub}_{2} X$ is the composite $X \stackrel{\Delta}{\longrightarrow} X \times X \longrightarrow \mathrm{SP}^{2} X \cong \operatorname{Sub}_{2} X$, where $\Delta$ is the diagonal, while $j_{2}:: X \hookrightarrow \mathrm{SP}^{2} X$ is the basepoint inclusion.

Example 2.3. When $X=S^{1}, \mathrm{SP}^{2}\left(S^{1}\right)$ is the closed Möbius band. If we view this band as a square with two sides identified along opposite orientations, then $S^{1}=$ $\mathrm{SP}^{1}\left(S^{1}\right) \hookrightarrow \mathrm{SP}^{2}\left(S^{1}\right)$ embeds into this band as an edge (see figures on p. 1124 of [23]).

Hence this embedding is homotopic to the embedding of an equator, and so $\overline{\mathrm{SP}}^{2}\left(S^{1}\right)$ is contractible. On the other hand, $S^{1}=\operatorname{Sub}_{1}\left(S^{1}\right)$ embeds into $\operatorname{Sub}_{2}\left(S^{1}\right)=\operatorname{SP}^{2}\left(S^{1}\right)$ as the diagonal $x \mapsto\{x, x\}=[x, x]$, which is the boundary of the Möbius band, and so $\overline{\operatorname{Sub}}_{2}\left(S^{1}\right)=\mathbb{R} P^{2}$.

Example 2.4. When $X=S^{2}, \mathrm{SP}^{2}\left(S^{2}\right)$ is the complex projective plane $\mathbb{P}^{2}, \mathrm{SP}^{1}\left(S^{2}\right)=$ $\mathbb{P}^{1}$ is a hyperplane, and $\overline{\mathrm{SP}}^{2}\left(S^{2}\right)=S^{4}$. On the other hand, $\overline{\operatorname{Sub}}_{2}\left(S^{2}\right)$ has the following description: Write $\mathbb{P}^{1}$ for $\mathbb{C} \cup\{\infty\}$. Then $\overline{\operatorname{Sub}}_{2}\left(S^{2}\right)$ is the quotient of $\mathbb{P}^{2}$ by the image of the Veronese embedding $\mathbb{P}^{1} \longrightarrow \mathbb{P}^{2}, z \mapsto\left[z^{2}:-2 z: 1\right], \infty \mapsto[1: 0: 0]$. To see this, identify $\operatorname{SP}^{n}(\mathbb{C})$ with $\mathbb{C}^{n}$ by sending $\left(z_{1}, \ldots, z_{n}\right)$ to the coefficients of the polynomial $\left(x-z_{1}\right) \cdots\left(x-z_{n}\right)$. This extends to the compactifications to give an identification of $\operatorname{SP}^{n}\left(S^{2}\right)$ with $\mathbb{P}^{n}([\mathbf{1 0}$, Chapter 4$])$. When $n=1,(z, z)$ is mapped to the coefficients of $(x-z)(x-z)$, that is to $\left(z^{2},-2 z\right)$. Note that the diagonal $S^{2} \longrightarrow \mathrm{SP}^{2}\left(S^{2}\right)=\mathbb{P}^{2}$ is multiplication by 2 on the level of $H_{2}$ so that, in particular, $H_{4}\left(\overline{\operatorname{Sub}}_{2}\left(S^{2}\right)\right)=\mathbb{Z}$, $H_{2}\left(\overline{\operatorname{Sub}}_{2}\left(S^{2}\right)\right)=\mathbb{Z}_{2}$, and all other reduced homology groups are zero.

\section{Cell decomposition}

If $X$ is a simplicial complex, then there is a standard way to pick a $\mathfrak{S}_{n}$-equivariant simplicial decomposition for the product $X^{n}$ so that the quotient map $X^{n} \longrightarrow \operatorname{SP}^{n} X$ induces a cellular structure on $\mathrm{SP}^{n} X$. We argue that this same cellular structure descends to a cell structure on $\mathrm{Sub}_{n} X$. The construction of this cell structure for the symmetric products is fairly classical $[\mathbf{1 4}, \mathbf{1 8}]$. The following is a review and slight expansion: 
Proposition 3.1. Let $X$ be a simplicial complex. For $n \geqslant 1$, there exist cellular decompositions for $X^{n}, \mathrm{SP}^{n} X$ and $\mathrm{Sub}_{n} X$ so that all of the quotient maps

$$
X^{n} \rightarrow \mathrm{SP}^{n} X \rightarrow \operatorname{Sub}_{n} X
$$

and the concatenation pairings + are cellular

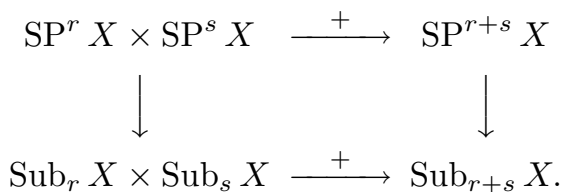

Furthermore, the subspaces $\Delta^{n}, \mathrm{SP}^{n-1} X \subset \mathrm{SP}^{n} X$ and $\operatorname{Sub}_{n-1} X \subset \operatorname{Sub}_{n} X$ are subcomplexes.

Proof. Both $\mathrm{SP}^{n} X$ and $\mathrm{Sub}_{n} X$ are obtained from $X^{n}$ via identifications. If for some simplicial (hence cellular) structure on $X^{n}$, derived from that on $X$, these identifications become simplicial (i.e. they identify simplices to simplices), then the quotients will have a cellular structure and the corresponding quotient maps will be cellular with respect to these structures.

As we know, one obtains a nice and natural $\mathfrak{S}_{n}$-equivariant simplicial structure on the product if one works with ordered simplicial complexes $[\mathbf{8}, \mathbf{1 4}, \mathbf{1 8}]$. We write $X$. for the abstract simplicial (i.e. triangulated) complex of which $X$ is the realization. So we assume $X$. to be endowed with a partial ordering on its vertices which restricts to a total ordering on each simplex. Let $\prec$ be that ordering. A point $w=\left(v_{1}, \ldots, v_{n}\right)$ is a vertex in $X_{\bullet}^{n}$ if and only if $v_{i}$ is a vertex of $X_{\bullet}$. Different vertices

$$
w_{0}=\left(v_{01}, v_{02}, \ldots, v_{0 n}\right), \ldots, w_{k}=\left(v_{k 1}, v_{k 2}, \ldots, v_{k n}\right)
$$

span a $k$-simplex in $X_{\bullet}^{n}$ if, and only if, for each $i$, the $k+1$ vertices $v_{0 i}, v_{1 i}, \ldots, v_{k i}$ are contained in a simplex of $X$ and $v_{0 i} \prec v_{1 i} \prec \cdots \prec v_{k i}$. We write $\varpi:=\left[w_{0}, \ldots, w_{k}\right]$ for such a simplex.

The permutation action of $\tau \in \mathfrak{S}_{n}$ on $\varpi=\left[w_{0}, \ldots, w_{k}\right]$ is given by

$$
\tau \varpi=\left[\tau w_{0}, \ldots, \tau w_{n}\right] .
$$

This is a well-defined simplex since the factors of each vertex

$$
w_{j}=\left(v_{j_{1} 1}, v_{j_{2} 2}, \ldots, v_{j_{n} n}\right)
$$

are permuted simultaneously according to $\tau$, and hence the order $\prec$ is preserved. The permutation action is then simplicial and $\mathrm{SP}^{n} X$ inherits a $\mathrm{CW}$-structure by passing to the quotient.

Fact 1. If a point $p:=\left(x_{1}, x_{2}, \ldots, x_{n}\right) \in X^{n}$ is such that $x_{i_{1}}=x_{i_{2}}=\cdots=x_{i_{r}}$, then $p$ lies in some $k$-simplex $\varpi$ whose vertices $\left[w_{0}, \ldots, w_{k}\right]$ are such that $v_{j i_{1}}=v_{j i_{2}}=\cdots=$ $v_{j i_{r}}$ for $j=0, \ldots, k$. This implies that the fat diagonal is a simplicial subcomplex. It also implies that any permutation that fixes such a point $p$ must fix the vertices of the simplex it lies in and hences fixes it pointwise. In other words, if a permutation leaves a simplex invariant then it must fix it pointwise.

Fact 2. If $p=\left(x_{1}, x_{2}, \ldots, x_{n}\right) \in \varpi$ is a simplex with vertices $w_{0}, \ldots, w_{k}$ as in (5), and if $\pi: X^{n} \longrightarrow X^{i}$ is any projection, then $\pi(p)$ lies in the simplex with vertices 
$\pi\left(w_{0}\right), \ldots, \pi\left(w_{k}\right)$ (which may or may not be equal). For instance, $\pi(p):=\left(x_{1}, \ldots, x_{i}\right)$ lies in the simplex with vertices $\left(v_{01}, v_{02}, \ldots, v_{0 i}\right), \ldots,\left(v_{k 1}, v_{k 2}, \ldots, v_{k i}\right)$.

We are now in a position to see that $\operatorname{Sub}_{n} X$ is a CW-complex. Recall that $\operatorname{Sub}_{n} X=X^{n} / \sim$, where

$$
\left(x_{1}, \ldots, x_{n}\right) \sim\left(y_{1}, \ldots, y_{n}\right) \Longleftrightarrow\left\{x_{1}, \ldots, x_{n}\right\}=\left\{y_{1}, \ldots, y_{n}\right\} .
$$

Clearly, if $\left(x_{1}, \ldots, x_{n}\right) \sim\left(y_{1}, \ldots, y_{n}\right)$, then $\tau\left(x_{1}, \ldots, x_{n}\right) \sim \tau\left(y_{1}, \ldots, y_{n}\right)$ for $\tau \in \mathfrak{S}_{n}$. We wish to show that these identifications are simplicial. Let us argue through an example (the general case being identical). We have the identifications in $\operatorname{Sub}_{6} X$ :

$$
p:=(x, x, x, y, y, z) \sim(x, x, y, y, y, z)=: q .
$$

By using Fact 2 applied to the projection, skipping the third coordinate and then Fact 1, we can see that $p$ and $q$ lie in simplices with vertices of the form

$$
\left(v_{1}, v_{1}, ?, v_{2}, v_{2}, v_{3}\right) \text {. }
$$

By using Fact 1 again, $p$ lies in a simplex $\sigma_{p}$ with vertices of the form

$$
\left(v_{1}, v_{1}, v_{1}, v_{2}, v_{2}, v_{3}\right) \text {, }
$$

while $q$ lies in a simplex $\sigma_{q}$ with vertices of the form $\left(v_{1}, v_{1}, v_{2}, v_{2}, v_{2}, v_{3}\right)$. It follows that the identification (6) identifies vertices of $\sigma_{p}$ with vertices of $\sigma_{q}$, and hence identifies $\sigma_{p}$ with $\sigma_{q}$ as desired.

In conclusion, the quotient $\operatorname{Sub}_{n} X$ inherits a cellular structure and the composite

$$
X^{n} \stackrel{\pi}{\rightarrow} \mathrm{SP}^{n} X \stackrel{q}{\rightarrow} \operatorname{Sub}_{n} X
$$

is cellular. Since the pairing (4) is covered by $X^{r} \times X^{s} \longrightarrow X^{r+s}$, which is simplicial (by construction), and since the projections are cellular, the pairing (4) must be cellular.

Remark 3.2. We could have worked with simplicial sets instead [5]. Similarly, Mostovoy (private communication) indicates how to construct a simplicial set $\operatorname{Sub}_{n} X$ out of a simplicial set $X$ such that $\left|\operatorname{Sub}_{n} X\right|=\operatorname{Sub}_{n}|X|$. This approach will be further discussed in [12].

The following corollary is also obtained in [5].

Corollary 3.3. For $X$ a simplicial complex, $\mathrm{Sub}_{k} X$ has a $\mathrm{CW}$-decomposition with top cells in $k \operatorname{dim} X$, so that $H_{*}\left(\operatorname{Sub}_{k} X\right)=0$ for $*>k \operatorname{dim} X$.

We collect a couple more corollaries

Corollary 3.4. If $X$ is a d-dimensional complex with $d \geqslant 2$, then the quotient map $\mathrm{SP}^{n} X \rightarrow \operatorname{Sub}_{n} X$ induces a homology isomorphism in top dimension nd.

Proof. When $X$ is as in the hypothesis, $\operatorname{Sub}_{n-1} X$ is a codimension $d$ subcomplex of $\operatorname{Sub}_{n} X$ and since $d \geqslant 2, H_{n d}\left(\operatorname{Sub}_{n} X\right)=H_{n d}\left(\operatorname{Sub}_{n} X, \operatorname{Sub}_{n-1} X\right)$. On the other hand, Proposition 3.1 implies that $\Delta^{n} X$ is a codimension $d$ subcomplex of $\mathrm{SP}^{n} X$ so that $H_{n d}\left(\mathrm{SP}^{n} X\right) \cong H_{n d}\left(\mathrm{SP}^{n} X, \Delta^{n} X\right)$ as well. But according to diagram (2), we have the homeomorphism

$$
\mathrm{SP}^{n} X / \Delta^{n} X \cong \operatorname{Sub}_{n} X / \operatorname{Sub}_{n-1} X .
$$

Combining these facts yields the claim. 
Corollary 3.5. Both $\mathrm{SP}^{k} X$ and the fat diagonal $\Delta^{k} \subset \mathrm{SP}^{k} X$ have the same connectivity as $X$, and this is sharp.

Proof. If $X$ is an $r$-connected ordered simplicial complex, then $X$ admits a simplicial structure so that the $r$-skeleton $X_{r}$ is contractible in $X$ to some point $x_{0} \in X$. With such a simplicial decomposition we can consider Liao's induced decomposition $X_{\bullet}^{k}$ on $X^{k}$ and its $r$-skeleton $X_{r}^{k}$. Note that

$$
X_{r}^{k} \subset \bigcup_{i_{1}+\cdots+i_{k} \leqslant r} X_{i_{1}} \times X_{i_{2}} \times \cdots \times X_{i_{k}} \subset\left(X_{r}\right)^{k} .
$$

If $F: X_{r} \times I \longrightarrow X$ is a deformation of $X_{r}$ to $x_{0}$, then $F^{k}$ is a deformation of $\left(X_{r}\right)^{k}$; hence $X_{r}^{k}$, to $\left(x_{0}, \ldots, x_{0}\right)$ in $X^{k}$, and this deformation is $\mathfrak{S}_{k}$ equivariant. Since the $r$-skeleton of $\mathrm{SP}^{k} X$ is the $\mathfrak{S}_{k}$-quotient of $X_{r}^{k}$, it is then itself contractible in $\mathrm{SP}^{k} X$, and this proves the first claim. Similarly, the simplicial decomposition we have introduced on $X^{k}$ includes the fat diagonal $\Lambda^{k}$ as a subcomplex with $r$-skeleton $\Lambda_{r}^{k}:=\Lambda^{k} \cap X_{r}^{k}$. The deformation $F^{k}$ preserves the fat diagonal and so it restricts to $\Lambda^{k}$ and to an equivariant deformation $F^{k}: \Lambda_{r}^{k} \times I \longrightarrow \Lambda^{k}$. This means that the $r$-skeleton of $q\left(\Lambda^{k}\right)=: \Delta^{k} \subset \mathrm{SP}^{k} X$ is itself contractible in $\Delta^{k}$, and the second claim follows. This bound is sharp for symmetric products since when $X=S^{2}$, $\operatorname{SP}^{2}\left(S^{2}\right)=\mathbb{P}^{2}$. It is sharp for the fat diagonal as well since $\Delta^{3} X \cong X \times X$ has exactly the same connectivity of $X$.

\section{Connectivity}

As we have established in Corollary 2.2, finite subset spaces $\operatorname{Sub}_{n} X, n \geqslant 3$, are always simply connected. In this section we further relate the connectivity of $\operatorname{Sub}_{k} X$ to that of $X$. We first need the following useful result proved in [11]:

Theorem 4.1. If $X$ is r-connected with $r \geqslant 1$, then $\overline{\mathrm{SP}}^{n} X$ is $2 n+r$-2-connected.

Example 5.7 shows that $\overline{\mathrm{SP}}^{2}\left(S^{k}\right)$ is $k+1$-connected as asserted. Note that

$$
\overline{\mathrm{SP}}^{2}\left(S^{2}\right)=S^{4}
$$

is 3-connected, so Theorem 4.1 is sharp.

Corollary 4.2 ([18, Corollary 4.7$])$. If $X$ is $r$-connected, $r \geqslant 1$, then

$$
H_{*}(X) \cong H_{*}\left(\mathrm{SP}^{n} X\right)
$$

for $* \leqslant r+2$. This isomorphism is induced by the map $j_{n}$ adjoining the basepoint.

Proof. We give a short proof based on Theorem 4.1. By Steenrod's homological splitting $[\mathbf{1 8}]$

$$
H_{*}\left(\mathrm{SP}^{n} X\right) \cong \bigoplus_{k=1}^{n} H_{*}\left(\mathrm{SP}^{k} X, \mathrm{SP}^{k-1} X\right)=\bigoplus_{k=2}^{n} \tilde{H}_{*}\left(\overline{\mathrm{SP}}^{k} X\right) \oplus H_{*}(X)
$$

with $\mathrm{SP}^{0} X=\emptyset$, but $\tilde{H}_{*}\left(\overline{\mathrm{SP}}^{k} X\right)=0$ for $* \leqslant 2 k+r-2$. The result follows. 
Remark 4.3. Note that Corollary 4.2 cannot be improved to $r=0$ (i.e. $X$-connected). It fails already for the wedge $X=S^{1} \vee S^{1}$ and $n=2$ since $\operatorname{SP}^{2}\left(S^{1} \vee S^{1}\right) \simeq S^{1} \times S^{1}$ (see [13]) and hence $H_{2}\left(\operatorname{SP}^{2}\left(S^{1} \vee S^{1}\right)\right) \neq H_{2}\left(S^{1} \vee S^{1}\right)$. Note also that (7) implies that $H_{*}(X)$ embeds into $H_{*}\left(\mathrm{SP}^{n} X\right)$ for all $n \geqslant 1$, a fact we will find useful below.

Proposition 4.4. Suppose $X$ is $r$-connected, $r \geqslant 1$. Then $\operatorname{Sub}_{k} X$ is $r+1$-connected whenever $k \geqslant 3$.

Proof. Write $x_{0} \in X$ for the basepoint and assume $k \geqslant 3$. Remember that the $\operatorname{Sub}_{k} X$ are simply connected for $k \geqslant 3$ (Corollary 2.2) so by the Hurewicz theorem if they have trivial homology up to degree $r+1$, then they are connected up to that level. We will now show by induction that $H_{*}\left(\operatorname{Sub}_{k} X\right)=0$ for $* \leqslant r+1$. The first step is to show that $H_{*}\left(\mathrm{SP}^{k} X, \Delta^{k}\right)=H_{*}\left(\operatorname{Sub}_{k} X, \operatorname{Sub}_{k-1} X\right)=0$ for $* \leqslant r+1$. We write $i: \Delta^{k} \hookrightarrow \mathrm{SP}^{k} X$ for the inclusion.

From the fact that $\Delta^{k}$ and $\mathrm{SP}^{k} X$ have the same connectivity as $X$ (Corollary 3.5), their homology vanishes up to degree $r$ which implies similarly that the relative groups are trivial up to that degree. On the other hand, $X$ embeds in $\Delta^{k}$ via $x \mapsto\left[x, x_{0}, \ldots, x_{0}\right]$ (this is a well-defined map since $k \geqslant 3$ ) and, since the composite $j_{k}: X \longrightarrow \boldsymbol{\Delta}^{\mathbf{k}} \stackrel{i}{\rightarrow} \mathrm{SP}^{k} X$ is an isomorphism on $H_{r+1}$ (Corollary 4.2 ), we see that the map $i_{*}: H_{r+1}\left(\boldsymbol{\Delta}^{k}\right) \longrightarrow H_{r+1}\left(\mathrm{SP}^{k} X\right)$ is surjective. Hence, $H_{r+1}\left(\mathrm{SP}^{k} X, \boldsymbol{\Delta}^{k}\right)=0$.

Now since $0=H_{*}\left(\mathrm{SP}^{k} X, \Delta^{k}\right)=H_{*}\left(\operatorname{Sub}_{k} X, \operatorname{Sub}_{k-1} X\right)$ for $* \leqslant r+1$, it follows that

$$
H_{*}\left(\operatorname{Sub}_{k-1} X\right) \cong H_{*}\left(\operatorname{Sub}_{k} X\right) \quad \text { for } * \leqslant r
$$

and that

$$
H_{r+1}\left(\operatorname{Sub}_{k-1} X\right) \rightarrow H_{r+1}\left(\operatorname{Sub}_{k} X\right) \quad \text { is surjective. }
$$

So if we prove that $H_{*}\left(\mathrm{Sub}_{3} X\right)=0$ for $* \leqslant r+1$, then by induction we will have proved our claim.

Consider the homology long exact sequences for

$$
\left(\mathrm{Sub}_{3} X, \mathrm{Sub}_{2} X\right) \quad \text { and } \quad\left(\mathrm{SP}^{3} X, \Delta^{3} X\right),
$$

where again we identify $\Delta^{3} X$ with $X \times X$. We obtain commutative diagrams

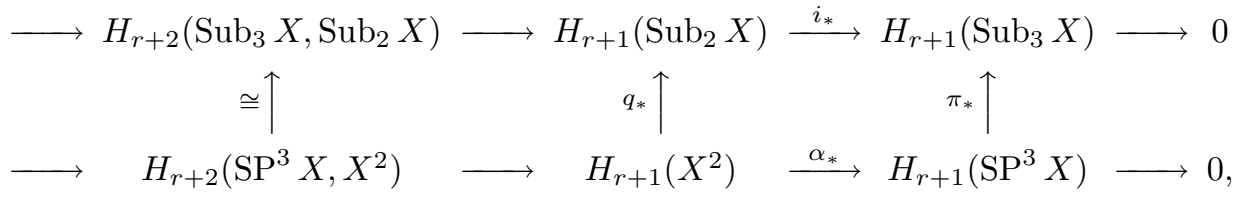

where $\alpha(x, y)=x^{2} y$ and $\pi: \mathrm{SP}^{3} X \longrightarrow \mathrm{Sub}_{3} X$ is the quotient map. We want to show that $i_{*}=0$ so that by exactness $H_{r+1}\left(\operatorname{Sub}_{3} X\right)=0$. Now $q_{*}$ is surjective since the composite

$$
X \longrightarrow X \times\left\{x_{0}\right\} \hookrightarrow X \times X \longrightarrow \mathrm{SP}^{2} X=\mathrm{Sub}_{2} X
$$

induces an isomorphism on $H_{r+1}$ by Corollary 4.2. Showing that $i_{*}=0$ comes down, therefore, to showing that $\pi_{*} \circ \alpha_{*}=0$. But note that for $r \geqslant 1$, which is the connectivity of $X$, classes in $H_{r+1}(X \times X)$ are necessarily spherical and we have the 
following commutative diagram:

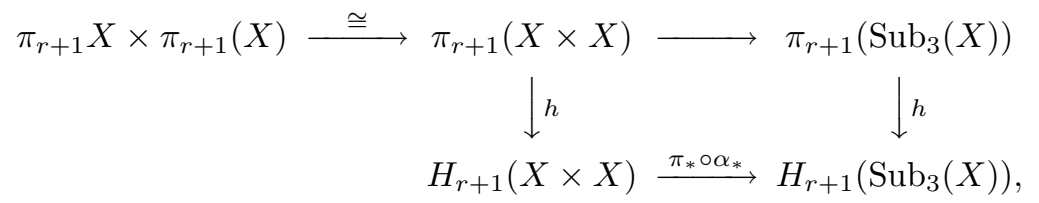

where $h$ is the Hurewicz homomorphism. The top map is trivial since when restricted to each factor $\pi_{r+1}(X)$ it is trivial according to the useful Theorem 5.1 below (or to Corollary 5.2). Since $h$ is surjective, $\pi_{*} \circ \alpha_{*}=0$ and $H_{r+1}\left(\operatorname{Sub}_{3} X\right)=0$ as desired.

\section{The three-fold finite subset space}

There are many subtle points that come up in the study of finite subset spaces. We illustrate several of them through the study of the pair ( $\left.\mathrm{Sub}_{3} X, X\right)$. The three-fold subset space has been studied in $[\mathbf{1 7}, \mathbf{1 9}, \mathbf{2 3}]$ for the case of the circle and in $[\mathbf{2 4}]$ for topological surfaces.

Again all spaces below are assumed to be connected. We say a map is weakly contractible (or weakly trivial) if it induces the trivial map on all homotopy groups. The following is based on a cute argument well explained in $[\mathbf{9}]$ or $([\mathbf{3}, \S 3.4])$.

Theorem $5.1([9]) . \operatorname{Sub}_{k}(X)$ is weakly contractible in $\operatorname{Sub}_{2 k+1}(X)$.

Caveat 1. A map $f: A \longrightarrow Y$ being weakly contractible does not generally imply that $f$ is null homotopic. Indeed let $T$ be the torus and consider the projection $T \longrightarrow S^{2}$ which collapses the one-skeleton. Then this map induces an isomorphism on $H_{2}$ but is trivial on homotopy groups since $T=K\left(\mathbb{Z}^{2}, 1\right)$. Of course, if $A=S^{k}$ is a sphere, then "weakly trivial" and "null-homotopic" are the same since the map $A \longrightarrow Y$ represents the zero element in $\pi_{k} Y$. For example, in ([6, Lemma 3]), the authors explicitly construct an extension of the inclusion $S^{n} \hookrightarrow \operatorname{Sub}_{3}\left(S^{n}\right)$ to the disk $B^{n+1} \longrightarrow \operatorname{Sub}_{3}\left(S^{n}\right), \partial B^{n+1}=S^{n}$. This section argues that this implication does not generally hold for non-suspensions.

Caveat 2. When comparing symmetric products to finite subset spaces, one has to watch out for the fact that the basepoint inclusion $\mathrm{SP}^{k}(X) \longrightarrow \mathrm{SP}^{k+1}(X)$ does not commute via the projection maps with the inclusion $\operatorname{Sub}_{k}(X) \longrightarrow \operatorname{Sub}_{k+1}(X)$. This has already been pointed out in Example 2.3 and is further illustrated in the corollary below.

Corollary 5.2. The composite

$$
S P^{k}(X) \longrightarrow S P^{2 k+1}(X) \longrightarrow \operatorname{Sub}_{2 k+1}(X)
$$

is weakly trivial.

Proof. This map is equivalent to the composite

$$
\operatorname{SP}^{k}(X) \longrightarrow \operatorname{Sub}_{k}(X) \stackrel{\mu}{\longrightarrow} \operatorname{Sub}_{k+1}\left(X, x_{0}\right) \hookrightarrow \operatorname{Sub}_{2 k+1}(X),
$$

where $\mu\left(\left\{x_{1}, \ldots, x_{k}\right\}\right)=\left\{x_{0}, x_{1}, \ldots, x_{k}\right\}, x_{0}$ is the basepoint of $X$ and $\operatorname{Sub}_{k+1}\left(X, x_{0}\right)$ is the subspace of $\operatorname{Sub}_{k+1}(X)$ of all subsets containing this basepoint. Note that $\mu$ is 
not an embedding as pointed out in [24] but is one-to-one away from the fat diagonal. The key point here is again $([\mathbf{9}$, Theorem 4.1]) which asserts that the inclusion

$$
\operatorname{Sub}_{k+1}\left(X, x_{0}\right) \hookrightarrow \operatorname{Sub}_{2 k+1}\left(X, x_{0}\right)
$$

is weakly contractible. This in turn implies that the last map in (8) is weakly trivial as well and the claim follows.

Caveat 3. For $n \geqslant 2$, one can embed $X \hookrightarrow \operatorname{Sub}_{n}(X)$ in several ways. There is of course the natural inclusion $j$ giving $X$ as the subspace of singletons. There is also, for any choice of $x_{0} \in X$, the embedding $j_{x_{0}}: x \mapsto\left\{x, x_{0}\right\}$. Any two such embeddings for different choices of $x_{0}$ are equivalent when $X$ is path-connected (any choice of a path between $x_{0}$ and $x_{0}^{\prime}$ gives a homotopy between $j_{x_{0}}$ and $\left.j_{x_{0}^{\prime}}\right)$. It turns out, however, that $j$ and $j_{x_{0}}$ are fundamentally different. The simplest example was already pointed out for $S^{1}$, where $\operatorname{Sub}_{2}\left(S^{1}\right)$ was the Möbius band with $j$ being the embedding of the boundary circle while $j_{x_{0}}$ is the embedding of an equator.

One might ask the question whether it is true that $j$ is null-homotopic if and only if $j_{x_{0}}$ is null-homotopic? This is at least true for suspensions as the next lemma illustrates.

Recall that a co- $H$ space $X$ is a space whose diagonal map factors up to homotopy through the wedge; that is there exists a $\delta$ such that the composite

$$
X \stackrel{\delta}{\rightarrow} X \vee X \hookrightarrow X \times X
$$

is homotopic to the diagonal $\Delta: X \longrightarrow X \times X, x \mapsto(x, x)$. A cogroup $X$ is a co- $H$ space that is co-associative with a homotopy inverse. This latter condition means there is a map $c: X \longrightarrow X$ such that $X \stackrel{\delta}{\rightarrow} X \vee X \stackrel{\nabla(c \vee 1)}{\longrightarrow} X$ is null-homotopic. This is in fact the definition of a left inverse but it implies the existence of a right inverse as well [2]. If $X$ is a cogroup, then for every based space $Y$, the set of based homotopy classes of based maps $[X, Y]$ is a group. The suspension of a space is a cogroup and there exist several interesting cogroups that are not suspensions $([\mathbf{2}, \S 4])$.

Write $j_{x_{0}}: X \hookrightarrow \operatorname{Sub}_{3}\left(X, x_{0}\right)$ for the map $x \mapsto\left\{x, x_{0}\right\}$. Its continuation to $\operatorname{Sub}_{3}(X)$ is also written $j_{x_{0}}$.

Lemma 5.3. Suppose $X$ is a cogroup. Then the embeddings $j_{x_{0}}: X \hookrightarrow \operatorname{Sub}_{3}\left(X, x_{0}\right)$ and $j: X \hookrightarrow \operatorname{Sub}_{3}(X)$ are null-homotopic.

Proof. The argument in [9] extends to this situation. We deal with $j_{x_{0}}$ first. This is a based map at $x_{0}$. Its homotopy class $\left[j_{x_{0}}\right]$ lives in the group $G=\left[X, \operatorname{Sub}_{3}\left(X, x_{0}\right)\right]$. The following composite is checked to be again $j_{x_{0}}$ :

$$
j_{x_{0}}: X \stackrel{\Delta}{\longrightarrow} X \times X \stackrel{j_{x_{0}}+j_{x_{0}}}{\longrightarrow} \operatorname{Sub}_{3}\left(X, x_{0}\right) .
$$

This factors up to homotopy through the wedge

$$
\iota: X \stackrel{\delta}{\rightarrow} X \vee X \stackrel{j_{x_{0}} \vee j_{x_{0}}}{\longrightarrow} \operatorname{Sub}_{3}\left(X, x_{0}\right) .
$$

Of course $[\iota]=\left[j_{x_{0}}\right]$, but observe that $[\iota]=2\left[j_{x_{0}}\right]$ by definition of the additive structure of $G$. This means that $\left[j_{x_{0}}\right]=2\left[j_{x_{0}}\right]$; thus $\left[j_{x_{0}}\right]=0$ and $j_{x_{0}}$ is trivial (through a homotopy fixing $x_{0}$ ) 
Let us now apply this to the inclusion $j: X \hookrightarrow \operatorname{Sub}_{3}(X)$ which is assumed to be based at $x_{0}$. We also denote the composite $X \stackrel{j_{x_{0}}}{\longrightarrow} \operatorname{Sub}_{3}\left(X, x_{0}\right) \longrightarrow \operatorname{Sub}_{3} X$ by $j_{x_{0}}$. Using the co- $H$ structure as before, we get the homotopy commutative diagram

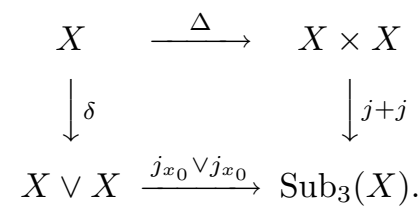

Since $j_{x_{0}}$ was just shown to be null homotopic, then so is $j=(j+j) \circ \Delta$.

Let us now turn to the second part of Theorem 1.2.

\subsection{The space $\operatorname{Sub}_{3}\left(X, x_{0}\right)$}

The preceding discussion shows the usefulness of looking at the based finite subset space $\operatorname{Sub}_{n}\left(X, x_{0}\right)$. We start with a key computation. Write $\Delta$ for the diagonal $X \longrightarrow$ $\mathrm{SP}^{2} X, x \mapsto[x, x]$, and identify the image of $j_{*}: H_{*}(X) \hookrightarrow H_{*}\left(\operatorname{SP}^{2}(X)\right)$ with $H_{*}(X)$ by the Steenrod homological splitting (7).

Lemma 5.4. Let $X$ be a compact cell complex. Then

$$
H_{*}\left(\operatorname{Sub}_{3}\left(X, x_{0}\right)\right)=H_{*}\left(\mathrm{SP}^{2} X\right) / I
$$

where $I$ is the submodule generated by $\Delta_{*} c-c, c \in H_{*}(X) \hookrightarrow H_{*}\left(\mathrm{SP}^{2} X\right)$.

Proof. Start with the map $\alpha: \operatorname{SP}^{2}(X) \longrightarrow \operatorname{Sub}_{3}\left(X, x_{0}\right),[x, y] \mapsto\left\{x, y, x_{0}\right\}$, which is surjective and generically one-to-one (i.e. one-to-one on the subspace of points $[x, y]$ with $x \neq y)$. Observe that $\alpha([x, x])=\alpha\left(\left[x, x_{0}\right]\right)$. This implies that $\operatorname{Sub}_{3}\left(X, x_{0}\right)$ is homeomorphic to the identification space

$$
\mathrm{SP}^{2}(X) / \sim, \quad[x, x] \sim\left[x, x_{0}\right], \quad \forall x \in X .
$$

In order to compute the homology of this quotient we will replace it with the following space:

$$
\begin{gathered}
W_{2}(X):=\mathrm{SP}^{2}(X) \sqcup X \times I / \sim, \\
{[x, x] \sim(x, 1), \quad\left[x, x_{0}\right] \sim(x, 0), \quad\left[x_{0}, x_{0}\right] \sim\left(x_{0}, t\right) .}
\end{gathered}
$$

It is not hard to see that (9) and (10) are homotopy equivalent. We can easily see that these spaces are homology equivalent as follows (this is enough for our purpose): There is a well-defined map

$$
g: W_{2}(X) \longrightarrow \operatorname{SP}^{2}(X) / \sim
$$

sending $[x, y] \mapsto[x, y],(x, t) \mapsto\left[x, x_{0}\right]$. The inverse image $g^{-1}([x, y])=[x, y]$ if $x \neq y$ and both points are different from $x_{0}$. The inverse image of $[x, x]$ or $\left[x, x_{0}\right]$ is an interval when $x \neq x_{0}$, hence contractible, and it is a point when $x=x_{0}$. In all cases, preimages under $g$ are acyclic and hence $g$ is a homology equivalence by the BegleVietoris theorem. The homology structure of $\operatorname{Sub}_{3}\left(X, x_{0}\right)$ can be made much more apparent using the form (10) and this is why we have introduced it.

Let $\left(C_{*}\left(\operatorname{SP}^{2}(X)\right), \partial\right)$ be a chain complex for $\operatorname{SP}^{2}(X)$ containing $C_{*}(X)$ as a subcomplex and for which the diagonal map $X \longrightarrow \mathrm{SP}^{2} X$ is cellular. Associate to 
$c \in C_{i}(X)$ a chain $|c|$ in degree $i+1$ representing $I \times c \in C_{i+1}(I \times X)$ if $c \neq x_{0}$ (the 0 -chain representing the basepoint). We write $\left|C_{*}(X)\right|$ for the set of all such chains. The geometry of our construction gives a chain complex for $W_{2}(X)$ as follows:

$$
C_{*}\left(W_{2}(X)\right)=C_{*}\left(\mathrm{SP}^{2}(X)\right) \oplus\left|C_{*}(X)\right|
$$

with boundary $d$ such that $d(c)=\partial c$ and

$$
d|c|=c-\Delta_{*}(c)-|\partial c| .
$$

This comes from the formula for the boundary of the product of two cells which is in general given by $\partial\left(\sigma_{1} \times \sigma_{2}\right)=\partial\left(\sigma_{1}\right) \times \sigma_{2}+(-1)^{\left|\sigma_{1}\right|} \sigma_{1} \times \partial\left(\sigma_{2}\right)$. We check indeed that $d \circ d=0$. To compute the homology we need to understand cycles and boundaries in this chain complex. Write a general element of (11) as $\alpha+|c|$. The boundary of this element is $\partial \alpha+c-\Delta_{*}(c)-|\partial c|$ and it is zero, if and only if, $\partial \alpha=\Delta_{*}(c)-c$ and $|\partial c|=0$. That is, if and only if, $c$ is a cycle and $\Delta_{*}(c)-c$ is a boundary. This means that in $H_{*}\left(\mathrm{SP}^{2}(C)\right), \Delta_{*}(c)=c$. We claim this is not possible unless $c=0$. Indeed, if $c$ is a positive dimensional (homology) class, then $\Delta_{*}(c)=c \otimes 1+\sum c^{\prime} \otimes c^{\prime \prime}+1 \otimes c$ in $H_{*}(X \times X)$ and hence in $H_{*}\left(\mathrm{SP}^{2}(C)\right), \Delta_{*}(c)=2 c+\sum c^{\prime} * c^{\prime \prime}$ where by definition $c^{\prime} * c^{\prime \prime}=q_{*}\left(c^{\prime} \otimes c^{\prime \prime}\right)$ and $q: X \times X \longrightarrow \mathrm{SP}^{2}(X)$ is the projection. This can never be equal to $c$ since $\sum c^{\prime} * c^{\prime \prime} \in H_{*}\left(\mathrm{SP}^{2} X, X\right)$.

To recapitulate, $\alpha+|c|$ is a cycle if and only if $\alpha$ is a cycle and $c=0$. The only cycles in $C_{*}\left(W_{2}(X)\right)$ are those that are already cycles in the first summand $C_{*}\left(\mathrm{SP}^{2}(X)\right)$. On the other hand, among these classes the only boundaries consist of boundaries in $C_{*}\left(\operatorname{SP}^{2}(X)\right)$ and those of the form $\Delta_{*}(c)-c$ with $c$ a cycle in $C_{*}(X)$ (in particular the only 0 -cycle is represented by $\left.x_{0}\right)$. This proves our claim.

Remark 5.5 (Added in revision). We could have noticed alternatively the existence of a pushout diagram

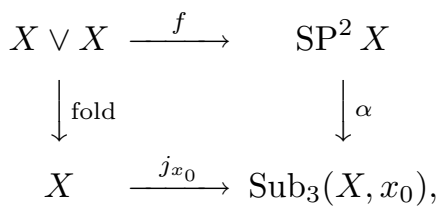

where $f\left(x, x_{0}\right)=[x, x]$ is the diagonal and $f\left(x_{0}, x\right)=\left[x, x_{0}\right]$. We can in fact deduce Lemma 5.4 from this pushout. We can also deduce that $\operatorname{Sub}_{3}\left(X, x_{0}\right)$ is simply connected if $X$ is. This useful fact we use to establish Proposition 5.6 next.

Note that Lemma 5.4 above says that $H_{*}\left(\operatorname{Sub}_{3}\left(X, x_{0}\right)\right)$ only depends on $H_{*}(X)$ and on its coproduct (i.e. on the cohomology of $X$ ). When $X$ is a suspension the situation becomes simpler. The following result is a nice combination of Lemmas 5.3 and 5.4.

Proposition 5.6. There is a homotopy equivalence $\operatorname{Sub}_{3}\left(\Sigma X, x_{0}\right) \simeq \overline{\mathrm{SP}}^{2}(\Sigma X)$.

Proof. When $X$ is a suspension, all classes are primitive so that $\Delta_{*}(c)=2 c$ for all $c \in H_{*}(X)$. Combining Steenrod's splitting (7),

$$
H_{*}\left(\mathrm{SP}^{2} X\right) \cong H_{*}(X) \oplus H_{*}\left(\mathrm{SP}^{2} X, X\right),
$$

with Lemma 5.4, we deduce immediately that $H_{*}\left(\operatorname{Sub}_{3}\left(\Sigma X, x_{0}\right)\right) \cong H_{*}\left(\overline{\mathrm{SP}}^{2}(\Sigma X)\right)$. Both spaces are simply connected (by Remark 5.5 and Theorem 4.1) and so it is 
enough to exhibit a map between them that induces this homology isomorphism. Consider the map $\alpha: \mathrm{SP}^{2}(\Sigma X) \longrightarrow \mathrm{Sub}_{3}\left(\Sigma X, x_{0}\right),[x, y] \mapsto\left\{x, y, x_{0}\right\}$ as in the proof of Lemma 5.4. Its restriction to $\Sigma X$ is null-homotopic according to Lemma 5.3 and hence it factors through the quotient $\overline{\mathrm{SP}}^{2}(\Sigma X) \longrightarrow \mathrm{Sub}_{3}\left(\Sigma X, x_{0}\right)$. By inspection of the proof of Lemma 5.4 we see that this map induces an isomorphism on homology.

Example 5.7. A description of $\overline{\mathrm{SP}}^{2}\left(S^{k}\right)$ is given in ([10, Example 4K.5]) from which we infer that

$$
\operatorname{Sub}_{3}\left(S^{k}, x_{0}\right) \simeq \Sigma^{k+1} \mathbb{R} P^{k-1}, \quad k \geqslant 1 .
$$

This generalizes the calculation in $[\mathbf{2 4}]$ that $\operatorname{Sub}_{3}\left(S^{2}, x_{0}\right) \simeq S^{4}$.

\subsection{Homology calculations}

We determine the homology of $\operatorname{Sub}_{3}\left(T, x_{0}\right)$ and $\operatorname{Sub}_{3}(T)$ where $T$ is the torus $S^{1} \times S^{1}$. Symmetric products of surfaces are studied in various places (see $[\mathbf{1 3}, \mathbf{2 4}]$ and references therein). Their homology is torsion free and hence particularly simple to describe. We will write $q: X^{n} \longrightarrow \mathrm{SP}^{n} X$ throughout for the quotient map and

$$
q_{*}\left(a_{1} \otimes \ldots \otimes a_{n}\right)=a_{1} * a_{2} * \cdots * a_{n}
$$

for its induced effect in homology. (Since our spaces are torsion free we identify $H_{*}(X \times Y)$ with $H_{*}(X) \otimes H_{*}(Y)$.)

Corollary 5.8. The inclusion $j: \operatorname{Sub}_{2}\left(T, x_{0}\right) \hookrightarrow \operatorname{Sub}_{3}\left(T, x_{0}\right)$ is essential.

Proof. We will show that $j_{*}$ is non-trivial on $H_{2}\left(\operatorname{Sub}_{2}\left(T, x_{0}\right)\right)=H_{2}(T)=\mathbb{Z}$. Here $H_{*}(T)$ is generated by $e_{1}, e_{2}$ in dimension one, and by the orientation class $[T]$ in dimension two. The groups $H_{*}\left(\mathrm{SP}^{2} T\right)$ are given as follows [13] (the generators are indicated between brackets):

$$
\tilde{H}_{*}\left(\mathrm{SP}^{2} T\right)=\left\{\begin{array}{lr}
\mathbb{Z}\left\{\gamma_{2}\right\}, & \operatorname{dim} 4 \\
\mathbb{Z}\left\{e_{1} *[T], e_{2} *[T]\right\}, & \operatorname{dim} 3 \\
\mathbb{Z}\left\{[T], e_{1} * e_{2}\right\}, & \operatorname{dim} 2 \\
\mathbb{Z}\left\{e_{1}, e_{2}\right\}, & \operatorname{dim} 1,
\end{array}\right.
$$

where $\gamma_{2}$ is the orientation class $\left[\mathrm{SP}^{2} T\right]\left(\mathrm{SP}^{2}(T)\right.$ is a compact complex surface). Then $[T] *[T]=2 \gamma_{2}$. Let $\Delta$ be the diagonal into the symmetric square

$$
X \stackrel{\Delta}{\rightarrow} X \times X \stackrel{q}{\rightarrow} \mathrm{SP}^{2}(X) .
$$

Since

$$
\begin{gathered}
\Delta_{*}([T])=[T] \otimes 1+e_{1} \otimes e_{2}-e_{2} \otimes e_{1}+1 \otimes[T], \\
q_{*}([T] \otimes 1)=q_{*}(1 \otimes[T])=[T]
\end{gathered}
$$

and

$$
q_{*}\left(e_{1} \otimes e_{2}\right)=-q_{*}\left(e_{2} \otimes e_{1}\right)=e_{1} * e_{2},
$$

we see that

$$
\Delta_{*}([T])=2[T]+2 e_{1} * e_{2} .
$$


We can consider the composite

$$
j_{x_{0}}: T \stackrel{\Delta}{\longrightarrow} \operatorname{SP}^{2} T \stackrel{\alpha}{\rightarrow} \operatorname{Sub}_{3}\left(T, x_{0}\right)=\mathrm{SP}^{2} T / \sim,
$$

where $\alpha$ is as in the proof of Lemma 5.4. According to Lemma 5.4, using the expression of the diagonal in (13), there are classes $a=\alpha_{*}[T], b=\alpha_{*}\left(e_{1} * e_{2}\right)$ with $a=-2 b \neq 0$. But $\left(j_{x_{0}}\right)_{*}[T]=(\alpha \circ \Delta)_{*}[T]=\alpha_{*}([T])=a$, and this is non-zero as desired.

Remark 5.9. We can of course complete the calculation of $H_{*}\left(\operatorname{Sub}_{3}\left(T, x_{0}\right)\right)$ from Lemma 5.4. Under $\alpha_{*}, e_{i} \mapsto 0$ (primitive classes map to 0 ), $e_{1} * e_{2} \mapsto b,[T] \mapsto a=$ $-2 b, e_{i} *[T] \mapsto c_{i}$, and $\gamma_{2} \mapsto d$, so that

$$
H_{1}=0, \quad H_{2}=\mathbb{Z}\{a\}, \quad H_{3}=\mathbb{Z}\left\{c_{1}, c_{2}\right\}, \quad H_{4}=\mathbb{Z}\{d\} .
$$

It is equally easy to write down the homology groups for $\operatorname{Sub}_{3}\left(S, x_{0}\right)$ for any genus $g \geqslant 1$ surface, orientable or not.

Next we analyze the inclusion $T \hookrightarrow \mathrm{Sub}_{3} T$ in the case of the torus (compare [24]). The starting point is the pushout (3) and the associated Mayer-Vietoris sequence

$$
\begin{aligned}
& \cdots \longrightarrow H_{*}(T \times T) \stackrel{q_{*} \oplus i_{*}}{\longrightarrow} H_{*}\left(\mathrm{SP}^{2} T\right) \oplus H_{*}\left(\mathrm{SP}^{3} T\right) \stackrel{g_{*}-\pi_{*}}{\longrightarrow} \\
& H_{*}\left(\mathrm{Sub}_{3} T\right) \longrightarrow H_{*-1}(T \times T) \longrightarrow \cdots,
\end{aligned}
$$

where $q: T \times T \longrightarrow \mathrm{SP}^{2} T$ is the quotient map, $i(x, y)=x^{2} y, g: \mathrm{SP}^{2} T \hookrightarrow \mathrm{Sub}_{3} T$ is the inclusion (here we have identified $\mathrm{SP}^{2} T$ with $\mathrm{Sub}_{2} T$ ) and $\pi: \mathrm{SP}^{3} T \longrightarrow \mathrm{Sub}_{3} T$ is the projection. We focus on degree 2 and follow $[\mathbf{1 3}]$ for the next computations.

We have $H_{2}(T \times T)=\mathbb{Z}^{2}$ generated by $[T] \otimes 1$ and $1 \otimes[T], H_{2}\left(\mathrm{SP}^{2} T\right)=\mathbb{Z}^{2}=$ $H_{2}\left(\mathrm{SP}^{3} T\right)$ generated by a class of the same name $[T]=q_{*}([T] \otimes 1)=q_{*}(1 \otimes[T])$ and by $e_{1} * e_{2} ;$ see (12). To describe the effect of $i_{*}$ we write it as a composite

$$
i: T \times T \stackrel{\Delta \times 1}{\longrightarrow} T \times T \times T \stackrel{q}{\rightarrow} \mathrm{SP}^{3} T .
$$

This gives $i_{*}([T] \otimes 1)=2[T]+2 e_{1} * e_{2}$ as in (13), while $i_{*}(1 \otimes[T])=[T]$. The MayerVietoris then looks like

$$
\begin{aligned}
\cdots \longrightarrow & \mathbb{Z}^{2} \stackrel{q_{*} \oplus i_{*}}{\longrightarrow} \mathbb{Z}^{2} \oplus \mathbb{Z}^{2} \stackrel{g_{*}-\pi_{*}}{\longrightarrow} H_{2}\left(\mathrm{Sub}_{3} T\right) \longrightarrow H_{1}(T \times T) \longrightarrow \cdots \\
(1,0) & \longmapsto((1,0),(2,2)) \\
(0,1) & \longmapsto((1,0),(1,0)) .
\end{aligned}
$$

This sequence is exact. Observe that the class $((2,2),(0,0))$ is not in the kernel of $g_{*}-\pi_{*}$ because it cannot be in the image of $q_{*} \oplus i_{*}$. This means that $g_{*}(2,2) \neq 0$. This is all we need to derive the non-nullity of the map $j: X \hookrightarrow \operatorname{Sub}_{3} X$.

Corollary 5.10. $j_{*}([T]) \neq 0$.

Proof. The inclusion $j$ is the composite

$$
j: T \stackrel{\Delta}{\longrightarrow} T \times T \stackrel{\pi}{\longrightarrow} \mathrm{SP}^{2} T \stackrel{g}{\rightarrow} \mathrm{Sub}_{3} T
$$

so that $j_{*}([T])=g_{*}(2,2)$, and this is non-trivial as asserted above. 


\section{The top dimension}

Using facts about orientability of configuration spaces of closed manifolds ([11] for example), we slightly elaborate on $[\mathbf{9}]$ and ([24, Theorem 3]).

Proposition 6.1. Suppose $M$ is a closed manifold of dimension $d \geqslant 2$. Then

$$
H_{n d}\left(S P^{n} M ; \mathbb{Z}\right)= \begin{cases}\mathbb{Z}, & \text { if } d \text { even and } M \text { orientable } \\ 0, & \text { if } d \text { odd or } M \text { non-orientable. }\end{cases}
$$

For mod-2 coefficients, $H_{n d}\left(S P^{n} M ; \mathbb{F}_{2}\right)=\mathbb{F}_{2}$. In all cases, the map

$$
H_{n d}\left(S P^{n} M\right) \longrightarrow H_{n d}\left(\operatorname{Sub}_{n} M\right)
$$

is an isomorphism (Corollary 3.4).

Proof. When $d=2$ the claim is immediate since, as is well known, $\mathrm{SP}^{n} M$ is a closed manifold (orientable if and only if $M$ is; see [26]). Generally our statement follows from the fact that $\mathrm{SP}^{n}(X)$ is an orbifold with codimension $>1$ singularities, and hence its top homology group is that of a manifold. More explicitly, in our case, let us denote by $B(M, n)$ the configuration space of finite sets of cardinality $n$ in $M$; that is

$$
B(M, n)=\mathrm{SP}^{n} M-\Delta^{n}=\operatorname{Sub}_{n} M-\operatorname{Sub}_{n-1} M,
$$

where $\Delta^{n}$ is the singular set consisting of tuples with at least one repeated entry (the image of the fat diagonal as defined in Section 2). By Poincaré duality suitably applied ([11, Lemma 3.5])

$$
H^{i}(B(M, n) ; \pm \mathbb{Z}) \cong H_{n d-i}\left(\mathrm{SP}^{n} M, \Delta^{n} ; \mathbb{Z}\right),
$$

where $\pm \mathbb{Z}$ is the orientation sheaf. By definition,

$$
H^{i}(B(M, n), \pm \mathbb{Z})=H^{i}\left(\operatorname{Hom}_{B r_{n}(M)}\left(C_{*}(\tilde{B}(M, n)), \mathbb{Z}\right)\right)
$$

where $B r_{n}(M)=\pi_{1}(B(M, n))$ is the braid group of $M, \tilde{B}(M, n)$ is the universal cover of $B(M, n)$ and the action of the class of a loop on $\mathbb{Z}$ is multiplication by \pm 1 according to whether the loop preserves or reverses orientation. It is known that $B(M, n)$ is orientable if and only if $M$ is orientable and even-dimensional ([11, Lemma 2.6]). That is, we can replace $\pm \mathbb{Z}$ by $\mathbb{Z}$ if $M$ is orientable and $d$ is even.

Since $\boldsymbol{\Delta}^{n}$ is a subcomplex of codimension $d$ in $\mathrm{SP}^{n} M$, we have

$$
H_{n d-i}\left(\mathrm{SP}^{n} M, \Delta^{n}\right) \cong H_{n d-i}\left(\mathrm{SP}^{n} M\right) \quad \text { for } \quad i<d-1 .
$$

In particular, for $i=0$ we obtain

$$
H^{0}(B(M, n) ; \pm \mathbb{Z}) \cong H_{n d}\left(\mathrm{SP}^{n} M ; \mathbb{Z}\right) .
$$

If $M$ is even-dimensional and orientable, then

$$
H^{0}(B(M, n) ; \pm \mathbb{Z}) \cong H^{0}(B(M, n) ; \mathbb{Z})=\mathbb{Z}
$$

since $B(M, n)$ is connected if $\operatorname{dim} M \geqslant 2$. If $\operatorname{dim} M$ is odd or $M$ is non-orientable, then $B(M, n)$ is not orientable and $H^{0}(B(M, n) ; \pm \mathbb{Z})=0$, because $H^{0}(B(M, n) ; \pm \mathbb{Z})$ is the subgroup $\left\{m \in \mathbb{Z} \mid g m=m, \forall g \in \mathbb{Z}\left[\pi_{1}(B(M, n)]\right\}\right.$. This establishes the claim for the 
symmetric products and hence for the finite subset spaces according to Corollary 3.4.

Example 6.2. For $k \geqslant 2$ we have $H_{2 k}\left(\mathrm{SP}^{2} S^{k}\right)=H_{2 k}\left(\overline{\mathrm{SP}}^{2} S^{k}\right)=H_{k-1}\left(\mathbb{R} P^{k-1}\right.$ ) (see Example 5.7) and this is $\mathbb{Z}$ or 0 depending on whether $k$ is even or odd as predicted by Proposition 6.1.

\subsection{The case of the circle}

When $M=S^{1}$, Proposition 6.1 is not true anymore since $\mathrm{SP}^{n} S^{1} \simeq S^{1}$ for all $n \geqslant 1$, while $\operatorname{Sub}_{n}\left(S^{1}\right)$ is either $S^{n}$ or $S^{n-1}$ depending on whether $n$ is odd or even $[\mathbf{1 5}, \mathbf{2 3}]$. In this case, it is still possible to explicitly describe the quotient map $\operatorname{SP}^{n}\left(S^{1}\right) \longrightarrow \operatorname{Sub}_{n}\left(S^{1}\right)$.

A beautiful theorem of Morton asserts that the multiplication map

$$
\mathrm{SP}^{n+1}\left(S^{1}\right) \longrightarrow S^{1}
$$

is an $n$-disc bundle $\eta_{n}$ over $S^{1}$ which is orientable if and only if $n$ is even [16]. A close scrutiny of Morton's proof shows that the sphere bundle associated to $\eta_{n}$ consists of the image of the fat diagonal $\boldsymbol{\Delta}^{n+1}$, i.e. the singular set. If $\operatorname{Th}\left(\eta_{n}\right)$ is the Thom space of $\eta_{n}$, then

$$
\operatorname{Th}\left(\eta_{n}\right)=\operatorname{SP}^{n+1}\left(S^{1}\right) / \Delta^{n+1}=\operatorname{Sub}_{n+1} S^{1} / \operatorname{Sub}_{n} S^{1} .
$$

Since $\eta_{n}$ is trivial when $n=2 k$ is even, it follows that

$$
\operatorname{Th}\left(\eta_{2 k}\right)=S^{2 k} \wedge S_{+}^{1}=S^{2 k+1} \vee S^{2 k} .
$$

However, as pointed out above, $\operatorname{Sub}_{2 k+1}\left(S^{1}\right) \simeq S^{2 k+1}$. The map

$$
\mathrm{SP}^{2 k+1}\left(S^{1}\right) \longrightarrow \operatorname{Sub}_{2 k+1}\left(S^{1}\right)
$$

factors through the Thom space (17) and the top cell maps to the top cell. Combining (16) and (17), it is immediate to see that

Lemma 6.3. The map $\operatorname{Th}\left(\eta_{2 k}\right) \longrightarrow \operatorname{Sub}_{2 k+1}\left(S^{1}\right)$, restricted to the first wedge summand in (17), induces a map $S^{2 k+1} \longrightarrow \operatorname{Sub}_{2 k+1}\left(S^{1}\right)$ which is a homotopy equivalence.

\section{Manifold structure}

In this last section we prove Theorem 1.3. We distinguish three cases: when the dimension of the manifold is $d>2, d=2$ or $d=1$.

Lemma 7.1. Suppose $X$ is a manifold of dimension $d>2$. Then $\operatorname{Sub}_{n} X$ is never a manifold if $n \geqslant 2$.

Proof. Consider the projection $X^{n} \longrightarrow \mathrm{Sub}_{n} X$ given by identifying tuples whose sets of coordinates are the same. This projection restricts to an $n$ ! regular covering between the complements $\pi_{n}: X^{n}-\Lambda^{n} \longrightarrow \operatorname{Sub}_{n} X-\operatorname{Sub}_{n-1} X$, where $\Lambda^{n}$ as before is the fat diagonal in $X^{n}$. Suppose $\operatorname{Sub}_{n} X$ is a manifold of dimension $n d$ (necessarily). Pick a point in $\operatorname{Sub}_{n-1} X$ and an open chart $U$ around it. Now $U \cong \mathbb{R}^{n d}$ and 
$Y=U \cap \mathrm{Sub}_{n-1} X$ is a closed subset in $U$. We can apply Alexander duality to the pair $(Y, U)$ and obtain

$$
H_{n d-i-1}(U-Y) \cong H^{i}(Y) .
$$

But $Y \subset \operatorname{Sub}_{n-1}(X)$ is an open subspace in a simplicial complex of dimension $(n-1) d$; therefore $H^{n d-2}(Y)=0$ (since $\left.d>2\right)$ and so $H_{1}(U-Y)=0$. We can now use an elementary observation of Mostovoy [17] to the effect that since $U-Y$ is covered by $\pi_{n}^{-1}(U-Y)$, a connected étale cover of degree $n$ !, then it is impossible for $H_{1}(U-Y)$ to be trivial since the monodromy gives a surjection $\pi_{1}(U-Y) \longrightarrow \mathfrak{S}_{n}$, and hence a non-trivial map $H_{1}(U-Y) \longrightarrow \mathbb{Z}_{2}$.

Theorem 2.4 of [26] shows that our Lemma 7.1 is valid if $d=2$ and $n>2$ as well. As opposed to the geometric approach of Wagner, we provide below a short homological proof of this result.

Lemma 7.2. Suppose $X$ is a closed topological surface. Then $\operatorname{Sub}_{n} X$ is a manifold if and only if $n=2$.

Proof. We will show that if $n \geqslant 3$, then $\operatorname{Sub}_{n}(X)$ cannot even have the homotopy type of a closed manifold by showing that it does not satisfy Poincaré duality. We rely on results of [13] that give a simple description of a CW-decomposition of a space $\widehat{\mathrm{SP}}^{n} X$ homotopy equivalent to $\mathrm{SP}^{n} X$ when $X$ is a two-dimensional complex. Since $X$ is a closed two-dimensional manifold, it has a cell structure of the form $X=\bigvee^{r} S^{1} \cup D^{2}$ where $D^{2}$ is a two-dimensional cell attached to a bouquet of circles. Each circle corresponds in the cellular chain complex for $\widehat{\mathrm{SP}}^{n} X$ to a one-dimensional cell generator $e_{i}, 1 \leqslant i \leqslant r$, while the two-dimensional cell is represented by $D$. This chain complex has a concatenation product $*: C_{*}\left(\widehat{\mathrm{SP}}^{r} X\right) \otimes C_{*}\left(\widehat{\mathrm{SP}}^{s} X\right) \longrightarrow C_{*}\left(\widehat{\mathrm{SP}}^{r+s} X\right)$ under which these cells map to product cells. The full cell complex for $\widehat{\mathrm{SP}}^{n} X$ is made up of all products of the form

$$
e_{i_{1}} * \cdots * e_{i_{\ell}} * \mathrm{SP}^{k} D, \quad i_{1}+\cdots+i_{\ell}+k \leqslant n,
$$

where $i_{r} \neq i_{s}$ if $r \neq s$, and where $\mathrm{SP}^{k} D$ is a $2 k$-dimensional cell represented geometrically by the $k$-th symmetric product of $D^{2}$. The boundary $\partial$ is a derivation and is completely determined on generators by $\partial e_{i}=0$ and $\partial \mathrm{SP}^{n} D=\partial D * \mathrm{SP}^{n-1} D$.

If $X=\bigvee^{r} S^{1} \cup D$ is a closed manifold, then in mod-2 homology, $\partial D=0$ (the top cell). This implies of course that $\partial \mathrm{SP}^{n} D=0$ (the top cell of $\mathrm{SP}^{n} X$ ), while $H_{2 n-1}\left(\mathrm{SP}^{n} X, \mathbb{Z}_{2}\right) \cong \mathbb{Z}_{2}^{r}$ with generators $e_{i} * \mathrm{SP}^{n-1} D$. This shows, in particular, that $H_{2 n-1}\left(\mathrm{SP}^{n} X ; \mathbb{Z}_{2}\right) \neq 0$ if $r \geqslant 1$, that is if $X$ is not the two sphere. Observe that this calculation is compatible with Theorem 2 of [24].

Now we know that $\operatorname{Sub}_{n} X$ is simply connected if $n \geqslant 3$. Suppose $\operatorname{Sub}_{n} X$ is a closed manifold, then by Poincaré duality, $H_{2 n-1}\left(\operatorname{Sub}_{n} X ; \mathbb{Z}_{2}\right)=H_{1}\left(\operatorname{Sub}_{n} X ; \mathbb{Z}_{2}\right)=0$. But recall the pushout diagram (2) and its associated Mayer-Vietoris exact sequence

$$
\begin{aligned}
H_{2 n-1}\left(\Delta_{n}\right) \longrightarrow H_{2 n-1}\left(\operatorname{Sub}_{n-1} X\right) & \oplus H_{2 n-1}\left(\mathrm{SP}^{n} X\right) \\
& \longrightarrow H_{2 n-1}\left(\operatorname{Sub}_{n} X\right) \longrightarrow H_{2 n-2}\left(\Delta_{n}\right) \longrightarrow \cdots .
\end{aligned}
$$

Since $\Delta_{n}$ and $\operatorname{Sub}_{n-1} X$ are $(2 n-2)$-dimensional subcomplexes of $\operatorname{Sub}_{n} X$, their 
homology in degree $2 n-1$ vanishes. The sequence above becomes

$$
0 \longrightarrow H_{2 n-1}\left(\mathrm{SP}^{n} X\right) \longrightarrow H_{2 n-1}\left(\operatorname{Sub}_{n} X\right) \longrightarrow H_{2 n-2}\left(\Delta_{n}\right) \longrightarrow \cdots
$$

and $H_{2 n-1}\left(\operatorname{SP}^{n} X\right)$ injects into $H_{2 n-1}\left(\operatorname{Sub}_{n} X\right)$. When $H_{1}(X) \neq 0$, that is when $X$ is not the sphere, $H_{2 n-1}\left(\operatorname{Sub}_{n} X\right)$ is non-trivial contradicting Poincaré duality.

We are left with the case $\operatorname{Sub}_{n}\left(S^{2}\right)$ and $n \geqslant 3$. Here we have to rely on a calculation of Tuffley $[\mathbf{2 4}]$ who shows that

$$
H_{2 n-2}\left(\operatorname{Sub}_{n}\left(S^{2}\right)\right)=\mathbb{Z} \oplus \mathbb{Z}_{n-1}
$$

But $\operatorname{Sub}_{n}\left(S^{2}\right)$ is 2-connected according to Theorem 1.1 and Poincaré duality is violated in this case as well.

Remark 7.3. A computation of the homology of $\operatorname{Sub}_{n}\left(S^{2}\right)$ for all $n$ and various field coefficients will appear in [12]. It is however straightforward using the Mayer-Vietoris sequence for the pushout (3) to show that

$$
\tilde{H}_{*}\left(\operatorname{Sub} 3 S^{2}\right) \cong \begin{cases}\mathbb{Z}, & *=6 \\ \mathbb{Z} \oplus \mathbb{Z}_{2}, & *=4\end{cases}
$$

Similar computations appear in $[\mathbf{5}, \mathbf{2 2}, \mathbf{2 4}]$.

Finally we address the case $d=1$. Write $I=[0,1], \dot{I}=(0,1)$. First of all $\mathrm{SP}^{n}(I) \cong$ $I^{n}$. In fact, this is precisely the $n$-simplex since any point of $\operatorname{SP}^{n}(I)$ can be written uniquely as an $n$-tuple $\left(x_{1}, \ldots, x_{n}\right)$ with $0 \leqslant x_{1} \leqslant \cdots \leqslant x_{n} \leqslant 1$. The quotient map $q_{2}: \mathrm{SP}^{2}(I) \longrightarrow \operatorname{Sub}_{2}(I)$ is a homeomorphism and hence every interior point of $\operatorname{Sub}_{2}(I)$ has a manifold neighborhood. The same for $n=3$ since $\operatorname{SP}^{3}(I)$ is the three simplex

$$
\left\{\left(x_{1}, x_{2}, x_{3}\right) \mid 0 \leqslant x_{1} \leqslant x_{2} \leqslant x_{3} \leqslant 1\right\}
$$

with four faces: $F_{1}:\left\{x_{1}=0\right\}, F_{2}:\left\{x_{1}=x_{2}\right\}, F_{3}:\left\{x_{2}=x_{3}\right\}$ and $F_{4}:\left\{x_{3}=1\right\}$, and the quotient map $q_{3}: \mathrm{SP}^{3}(I) \rightarrow \operatorname{Sub}_{3}(I)$ identifies the faces $F_{2}$ and $F_{3}$. Such an identification gives again $I^{3}$ and $\operatorname{Sub}_{3}(\dot{I})$ is this simplex with two faces removed [19]. For $n>3$, the corresponding map $q_{n}$ identifies various faces of the simplex $\mathrm{SP}^{n}(I)$ to obtain $\operatorname{Sub}_{n}(I)$, but this fails to give a manifold structure on the quotient for there are just too many "branches" that come together at a single point in the image of the boundary of this simplex. This is made precise below.

Lemma 7.4. $\operatorname{Sub}_{n}\left(S^{1}\right)$ is a closed manifold if and only if $n=1,3$.

Observe that if $n$ is even, then $\operatorname{Sub}_{n} S^{1}$ cannot be a closed manifold for a simple reason: no closed manifold of dimension $n$ can be homotopic to a sphere of dimension $n-1$.

Proof of Lemma 7.4 following [26, Theorem 2.3]. Let $M$ be a manifold and $D$ a disc neighborhood of a point $x \in M$. Then an open neighborhood of $x \in \operatorname{Sub}_{n}(M)$ is $\operatorname{Sub}_{n}(D)$. So if $\operatorname{Sub}_{n}(D)$ is not a manifold, then neither is $\operatorname{Sub}_{n}(M)$. To prove Lemma 7.4 we will argue as in $[\mathbf{2 6}]$ that $\operatorname{Sub}_{n}(\mathbb{R})$ is not a manifold for $n \geqslant 4$. 
For a metric space $X$ (with metric $d$ ), non-empty subsets $S, T \subset X$, and fixed elements $s \in S, t \in T$, we define

$$
\begin{aligned}
d(s, T) & =\inf \{d(s, t) \mid t \in T\}, \\
d(S, t) & =\inf \{d(s, t) \mid s \in S\} .
\end{aligned}
$$

Then the Hausdorff metric $D$ on $\operatorname{Sub}_{n}(X)$ is defined to be

$$
D(S, T):=\sup \{d(s, T), d(t, S) \mid s \in S, t \in T\} .
$$

Thus $D(S, T)<\epsilon$ means that each $s \in S$ is within an $\epsilon$-neighborhood of some point in $T$ and each $t \in T$ is within an $\epsilon$-neighborhood of some point in $S$.

We wish to show that $\operatorname{Sub}_{n}(\mathbb{R})$ for $n \geqslant 4$ is not homemorphic to $\mathbb{R}^{n}$. Pick $S=$ $\{1,2, \ldots, n-1\}$ in $\operatorname{Sub}_{n-1}(\mathbb{R})$ and for each $i$ consider the open set $C_{i}$ (in the Hausdorff metric) of all subsets $\left\{p_{1}, \ldots, p_{n-1}, q_{i}\right\} \in \operatorname{Sub}_{n}(\mathbb{R})$ such that $p_{j} \in\left(j-\frac{1}{2}, j+\frac{1}{2}\right)$ and $q_{i} \in\left(i-\frac{1}{2}, i+\frac{1}{2}\right)$. We then see that $C_{i}$ is the subset with one or two points in the $\frac{1}{2}$-neighborhood of $i$ and a single point in the $\frac{1}{2}$-neighborhood of $j$ for $i \neq j$. Note that $C_{i} \subset U$ where $U=\left\{T \in \operatorname{Sub}_{n}(\mathbb{R}) \mid D(S, T)<1 / 2\right\}$. Observe that

$$
C_{1}=\operatorname{Sub}_{2}\left(\frac{1}{2}, \frac{3}{2}\right) \times\left(\frac{3}{2}, \frac{5}{2}\right) \times \cdots \times\left(n-1-\frac{1}{2}, n-1+\frac{1}{2}\right) .
$$

This is an $n$-dimensional manifold with boundary $V=U \cap \operatorname{Sub}_{n-1}(\mathbb{R})$, and in fact one has

$$
C_{i}=\left\{T \in U: T \cap\left(i-\frac{1}{2}, i+\frac{1}{2}\right) \text { has } 1 \text { or } 2 \text { points }\right\} \cup V .
$$

Clearly $C_{1} \cup C_{2} \cup \cdots \cup C_{n-1}=U$ and, more importantly, all these open sets have a common boundary at $V$; i.e. $C_{i} \cap C_{j}=V$. If $n \geqslant 4$, we can choose at least three such $C_{i}$, say $C_{1}, C_{2}, C_{3}$. Then $C_{1} \cup C_{2}$ is an open $n$-dimensional manifold (union over the common boundary $V$ ). It must be contained in the interior of $\operatorname{Sub}_{n}(\mathbb{R})$ and hence must be open there if $\operatorname{Sub}_{n}(\mathbb{R})$ were to be an $n$-dimensional manifold. But $C_{1} \cup C_{2}$ is not open in $\operatorname{Sub}_{n}(\mathbb{R})$ since every neighborhood of $\{1,2, \ldots, n-1\}$ must meet $C_{3}-V$ which is disjoint from $C_{1} \cup C_{2}$ (i.e. "too many" branches come together at that point).

We conclude this paper with the following cute theorem of Bott, which is the most significant early result on the subject:

Corollary 7.5 (Bott). There is a homeomorphism $\operatorname{Sub}_{3}\left(S^{1}\right) \cong S^{3}$.

Proof. It has been known since Seifert that the Poincaré conjecture holds for Seifert manifolds; that is, if a Seifert 3-manifold is simply connected then it is homeomorphic to $S^{3} \cdot{ }^{1}$ Clearly $\operatorname{Sub}_{3}\left(S^{1}\right)$ is a Seifert manifold where the action of $S^{1}$ on a subset is by multiplication on elements of that subset. Since it is simply connected (Corollary 2.2), the claim follows. Note that the $S^{1}$-action has two exceptional fibers consisting of the orbits of $\{1,-1\}$ and $\left\{1, j, j^{2}\right\}$ where $j=e^{2 \pi i / 3}$ (compare [23]).

\footnotetext{
${ }^{1}$ We thank Peter Zvengrowski for reminding us of this fact.
} 


\section{References}

[1] S. Albeverio, Y. Kodratiev, and M. Rockner, Analysis and geometry on configuration spaces, J. Functional Analysis 154 (1998), no. 2, 444-500.

[2] M. Arkowitz, Co- $H$-spaces, Handbook of algebraic topology, North-Holland, Amsterdam (1995), 1143-1173.

[3] A. Beilinson and V. Drinfeld, Chiral algebras, AMS Colloq. Publications 51, Amer. Math. Soc., Providence, RI, 2004.

[4] K. Borsuk and S. Ulam, On symmetric products of spaces, Bull. Amer. Math. Soc. 37 (1931), no. 12, 875-882.

[5] R. Biro, The homotopy cyclic configuration spaces and the homotopy type of Kontsevich's orientation space, Stanford thesis (1994).

[6] D. Curtis and N. Nhu, Hyperspaces of finite subsets which are homeomorphic to $\aleph_{0}$-dimensional linear metric spaces, Topology Appl. 19 (1985), no. 3, 251-260.

[7] A. Dold, Homology of symmetric products and other functors of complexes, Ann. of Math. (2) 68 (1958), no. 1, 54-80.

[8] B. Dwyer, Classifying spaces and homology decompositions, in Homotopy theoretic methods in group cohomology, Advanced Courses in Math, CRM Barcelona, Birkhauser (2001).

[9] D. Handel, Some homotopy properties of spaces of finite subsets of topological spaces, Houston J. Math. 26 (2000), no. 4, 747-764.

[10] A. Hatcher, Algebraic topology, Cambridge University Press, Cambridge (2002).

[11] S. Kallel, Symmetric products, duality and homological dimension of configuration spaces, in Groups, homotopy and configuration spaces, Geometry and Topology Monographs 13 (2008), 499-527.

[12] S. Kallel, Finite subset spaces and a spectral sequence of Biro, work in progress.

[13] S. Kallel and P. Salvatore, Symmetric products of two dimensional complexes, Contemp. Math. 407 (2006), 147-161.

[14] S.D. Liao, On the topology of cyclic products of spheres, Transactions Amer. Math. Soc. 77 (1954), 520-551.

[15] R.J. Milgram and R.C. Penner, Riemann's moduli space and the symmetric groups, in Mapping class groups and moduli spaces of Riemann surfaces (Göttingen, 1991/Seattle, WA, 1991), Contemp. Math. 150 (1993), 247-290.

[16] H.R. Morton, Symmetric products of the circle, Proc. Cambridge Philos. Soc. 63 (1967), 349-352.

[17] J. Mostovoy, Geometry of truncated symmetric products and real roots of real polynomials, Bull. London Math. Soc. 30 (1998), no. 2, 159-165.

[18] M. Nakaoka, Cohomology of symmetric products, J. Institute of Polytechnics, Osaka City University 8 (1957), no. 2, 121-140.

[19] S. Rose, A hyperbolic approach to $\exp _{3}\left(S^{1}\right)$, arXiv:0708.2085.

[20] R. Seroul, Anneau de cohomologie entière et $K$ U-théorie d'un produit symétrique d'une surface de Riemann, Publ. Dép. Math. (Lyon) 9 (1972), 27-66. 
[21] P.A. Smith, Manifolds with abelian fundamental group, Annals of Math. (2) 37 (1936), no. 3, 526-532.

[22] W. Taamallah, thesis in progress, University of Tunis.

[23] C. Tuffley, Finite subset spaces of $S^{1}$, Algebraic Geom. Topol. 2 (2002), 11191145 .

[24] C. Tuffley, Finite subset spaces of graphs and punctured surfaces, Algebr. Geom. Topol. 3 (2003), 873-904.

[25] C. Tuffley, Connectivity of finite subset spaces of cell complexes, Pacific J. Math. 217 (2004), no. 1, 175-179.

[26] C. Wagner, Symmetric, cyclic and permutation products of manifolds, dissertationes mathematicae, Polska Akademia Nauk, Warszawa (1980).

Sadok Kallel sadok.kallel@math.univ-lille1.fr

Laboratoire Painlevé, Université des Sciences et Technologies de Lille, France

Denis Sjerve sjer@math.ubc.ca

Department of Mathematics, University of British Columbia, Vancouver, Canada 